\title{
Archive-Based Multiobjective Evolutionary Algorithm for Large-Scale EV Charging Station Energy Management
}

\author{
Wanxing Sheng, Qing Duan, Haoqing Wang ${ }^{D}$, Guanglin Sha, and Chunyan Ma $(\mathbb{D}$ \\ Power Distribution Technology Center, China Electric Power Research Institute Beijing, Beijing 100192, China \\ Correspondence should be addressed to Haoqing Wang; wangbecks@163.com and Chunyan Ma; machunyan@epri.sgcc.com.cn
}

Received 31 May 2021; Revised 24 June 2021; Accepted 30 July 2021; Published 3 September 2021

Academic Editor: Shi Cheng

Copyright (c) 2021 Wanxing Sheng et al. This is an open access article distributed under the Creative Commons Attribution License, which permits unrestricted use, distribution, and reproduction in any medium, provided the original work is properly cited.

\begin{abstract}
With the increase in renewable energy, improving the utilization rate of renewable energy is of great practical significance. The microgrid has been proved effective in addressing this issue. As a flexible load, electric vehicles are connected to the grid on a large scale, which will have an impact on the grid. In order to solve this problem, this paper proposes a microgrid energy management model for electric vehicle charging stations, which takes into account the economics of microgrid operation and the stability of grid operation. Subsequently, this paper proposes an evolutionary multiobjective optimization algorithm to deal with constraints. Finally, this paper verifies the effectiveness of the proposed model and algorithm through experiments.
\end{abstract}

\section{Introduction}

With the continuous development of the social economy, our demand for energy has greatly increased [1]. The traditional energy sources are mainly the combustion of fossil energy, such as thermal power generation and various equipment using gasoline as fuel, which have brought serious harm to the environment [2]. On the one hand, the current renewable energy construction has made remarkable achievements and the installed capacity of photovoltaic and wind turbines has continued to increase. However, because renewable energy has the characteristics of uncertainty and randomness, the situation of power curtailment is very prominent, a large amount of renewable energy production capacity is wasted, and the development of renewable energy is a serious problem [3]. On the other hand, with the change of domestic people's ideology and the strong support of the government, the number of new energy vehicles represented by electric vehicles has increased year by year, which has played a certain role in alleviating environmental pollution. However, a large number of electric vehicles connected to the grid will also bring challenges to the stability and safety of grid operation [4]. If a large number of electric vehicles are connected to the grid for disorderly charging, during the peak period of electricity consumption, especially in the summer and winter seasons, the demand for electricity will rise rapidly, which will bring huge challenges to the power supply capacity and power transmission [5]. There are hidden dangers to electricity safety; in the low electricity consumption valley, such as late at night, the electricity consumption is less, and the electricity from the power station is nowhere to be used, resulting in a waste of renewable energy. Therefore, reasonable arrangements for the orderly charging of electric vehicles are of great significance to maintaining the stability and safety of the grid operation.

Through the microgrid management system [6], the electric vehicle can be used as a dispatchable load; that is, after the EV is connected, the energy network EMS will take over the control process, allocate the charging power and charging time of the $\mathrm{EV}$, and manage the entire process during the charging process. Through supervision, the impact on the grid can be mitigated. In addition, consider the energy interaction between electric vehicles and the grid (Vehicle to Grid, V2G). After connecting to electric vehicles, electric vehicles will regard electric vehicles as a special mobile energy storage system (because electric vehicles require energy to reach a certain level at a specific time). The charging and discharging power and charging/discharging 
time of electric vehicles are allocated by the energy network EMS. Studying the impact of electric vehicles on the grid is the basis for formulating charging and discharging strategies. At present, the research on the access of electric vehicles is mainly divided into two aspects. On the one hand, as an electric vehicle as a load, its charging behavior affects the power grid; on the other hand, as an energy storage device, a reasonable configuration of electric vehicles has positive significance for power grid regulation.

The disorderly charging of a large number of electric vehicles has an impact on the grid. If the charging behavior of EVs is coordinated and optimized and charging is performed in an orderly manner, the load fluctuation of the system can be reduced [7]. To a large extent, the abovementioned negative effects are reduced; with the popularization of electric vehicles, charging stations came into being. The research on charging control of charging stations will play an important role in the rational use of renewable energy and alleviating the pressure on the power grid. Therefore, this article firstly models the electric vehicle charging station microgrid, fully taking into account the random arrival of electric vehicles, taking the economy of charging station microgrid operation, stabilizing the grid load, and enhancing the stability of grid operation as the optimization goals, and designing the multiobjective optimization algorithm solves the actual problem and verifies the correctness and effectiveness of the proposed model and algorithm through experiments.

The follow-up writing of this article is arranged as follows. Chapter 2 introduces the energy management model of the charging station microgrid. Chapter 3 introduces the evolutionary multiobjective optimization algorithm to solve this problem in detail. Chapter 4 verifies the proposed method with experiments.

\section{Charging Station Microgrid Model}

2.1. Objectives. The electric vehicle charging station microgrid model includes photovoltaic power generation systems, energy storage systems, user loads, and electric vehicles. This is a grid-connected microgrid that is physically connected to the large power grid. When the microgrid is in operation, the energy supplier mainly includes the large power grid and photovoltaic power generation system and the energy user includes user loads and electric vehicles; the energy storage system can be used as an energy consuming device or as an energy storage device. To make the operating load economy and stability principles of the entire microgrid, the orderly control of electric vehicle charging is the key.

For the operation planning and design of the hybrid renewable energy system, different optimization goals will bring different optimization results [8]. From the research status of the first part of the hybrid energy system, it can be seen that many goals can be considered in the optimal design of hybrid energy systems, and some consider only a single goal, and some consider multiple goals at the same time. Although these optimization goals have different names and meanings, they can be divided into three categories: economic goals, reliability goals, and environmental benefits goals. Economic goals are mainly indicators related to system cost, such as total system cost, system net present value cost, system capacity cost, and system annualized cost; reliability goals include total unmet load, unmet load ratio, and power supply missing rate; environmental benefit targets mainly refer to emission-related indicators such as annual carbon dioxide emissions, greenhouse gas emissions, and equivalent carbon dioxide emissions during the life cycle. These three types of goals represent three aspects that need to be considered during system planning and design. An optimal system configuration scheme should weigh these three goals into consideration.

Penalty fee for not reaching the expected charging capacity is as follows:

$$
C_{\mathrm{EV}}^{\text {punish }}=\rho_{\mathrm{EV}} \sum_{j=1}^{N_{\mathrm{EV}}}\left(E_{\mathrm{EV}, j}^{1}-E_{\mathrm{EV}, j}^{\text {leave }}\right) .
$$

The electric energy charge caused by charging is expressed as follows:

$$
C_{\text {charge }}=\sum_{i=1}^{N_{\text {charge }}} \sum_{k=0}^{T_{\text {end }}} P_{\text {rice }}(k) P_{\mathrm{EV}, i}(k) \Delta t .
$$

\subsection{Constraints}

2.2.1. Electric Vehicles. At any time, the arrival and departure of electric vehicles is a random process [9], and the probability of arrival and departure can be summarized and predicted based on historical data. In the predicted period, the arrival and departure of electric vehicles at a certain time can be approximately regarded as a Poisson distribution; that is, the probability of the number of electric vehicles arriving at any time is as follows:

$$
P(X=k)=\frac{\lambda(t)^{k}}{k !} e^{-\lambda}, \quad k=0,1, \ldots, N,
$$

where $\lambda(t)$ is the amount that changes with time and $N$ is the maximum vehicle arrival amount at time $t$. Based on statistical data and the arrival and departure Poisson distribution model of tourists, this paper uses the Monte Carlo method [10] to generate a series of electric vehicle arrival time, departure time, and remaining power data to solve the model.

As a flexible access load for electric vehicles, the charging time and power constraints are mainly considered. In this paper, $N_{\mathrm{EV}}$ represents the number of electric vehicles; $\delta_{\mathrm{EV}, i}$ represents the status of electric vehicles connected to the charging pile, where 1 means connected to the charging pile and 0 represents not connected; $\delta_{P, i}(k)$ represents the charging status of the electric vehicle; $\eta_{\mathrm{EV}}$ is the charging efficiency of the electric vehicle energy storage device; and $\varepsilon_{\mathrm{EV}}$ and $\Delta t$, respectively, represent the self-discharge energy loss of the electric vehicle energy storage device and the length of each cycle. Specifically, the staying time of electric vehicles is as follows: 


$$
T_{\mathrm{EV}, i}^{\mathrm{stay}} \in\left[T_{\mathrm{EV}, i}^{\text {arrive }}, T_{\mathrm{EV}, i}^{\text {leave }}\right] .
$$

The constraints of EV can be illustrated as follows:

$$
\begin{aligned}
& \sum_{k=T_{\mathrm{EV}, i}^{\text {arrive }}}^{T_{\mathrm{EV}, i}^{\text {leave }}-1} \delta_{\mathrm{EV}, i}(k)=T_{\mathrm{EV}, i}^{\text {leave }}-T_{\mathrm{EV}, i}^{\text {leave }}, \\
& \sum_{k=0}^{T_{\mathrm{EV}, i}^{\text {arre }}-1} \delta_{\mathrm{EV}, i}(k)+\sum_{k=T_{\mathrm{EV}, i}^{\text {leave }}-1}^{T_{\text {end }}} \delta_{E V, i}(k)=0, \\
& \delta_{P, i}(k) \leq \delta_{\mathrm{EV}, i}(k), \\
& \sum_{i=1}^{N_{\mathrm{EV}}} \delta_{\mathrm{EV}, i}(k) \leq N_{\mathrm{charge}}, \\
& P_{\mathrm{min}} \delta_{P, i}(k) \leq P_{\mathrm{EV}, i}(k) \leq P_{\mathrm{max}} \delta_{P, i}(k), \\
& \sum_{k=T_{\text {arrive }}}^{T_{\text {leave }}} \delta_{P, i}\left(\eta_{\mathrm{EV}, i}(k) P_{\max }-\varepsilon_{\mathrm{EV}}\right) \Delta t=E_{\mathrm{EV}, i}, \\
& E_{\mathrm{EV}, j}^{\text {leave }}=E_{\mathrm{EV}, j}^{0}+E_{\mathrm{EV}, j} .
\end{aligned}
$$

The above formula restricts whether the electric vehicle is in a state where it can be connected to the charging pile. The electric vehicle is in the state of being connected during the stay in the scenic spot and in the state of being inaccessible during the rest of the time. Equation (6) means that electric vehicles can only be connected to charging piles for charging when they are in an accessible state; equation (7) indicates that the number of electric vehicles currently connected to charging piles should be less than the number of existing charging piles; equation (8) is the maximum and minimum power constraints of charging piles; equation (9) is the dynamic constraint of electric vehicle charging energy, and the amount of charge that can be charged during the stay in the scenic spot; equation (10) represents the energy relationship of electric vehicles.

2.2.2. Energy Storage System. The energy storage system is one of the most flexible and directly controllable devices in the microgrid [11]. The position of the energy storage system is very unique. It can not only absorb excess electrical energy but also can be used as a backup power supply when the power is insufficient. Its main constraints include the maximum and minimum capacity limits of the energy storage system, the maximum charge and discharge limits, and charge and discharge energy transfer. The mathematical model of the electric vehicle charging station microgrid energy storage system can be described as follows:

$$
\begin{aligned}
& \delta_{\text {bess }}^{c}(k) P_{\text {bess }}^{c, \min } \leq P_{\text {bess }}^{c}(k) \leq \delta_{\text {bess }}^{c}(k) P_{\text {bess }}^{c, \max }, \\
& \delta_{\text {bess }}^{d}(k) P_{\text {bess }}^{d, \min } \leq P_{\text {bess }}^{d}(k) \leq \delta_{\text {bess }}^{d}(k) P_{\text {bess }}^{d, \max },
\end{aligned}
$$

$$
E_{\text {bess }}^{\min } \leq E_{\text {bess }}(k) \leq E_{\text {bess }}^{\max }
$$

$$
E_{\text {bess }}(k+1)=E_{\text {bess }}(k)+\left(\frac{\eta_{\text {bess }}^{c} P_{\text {bess }}^{c}(k)-P_{\text {bess }}^{d}(k)}{\eta_{\text {bess }}^{d}-\varepsilon_{\text {bess }}}\right) \Delta t \text {, }
$$

$$
\delta_{\text {bess }}^{c}(k)+\delta_{\text {bess }}^{d}(k) \leq 1 .
$$

Equations (11) and (12), respectively, constrain the charging and discharging power constraints of the energy storage system. When the energy storage system is not in the charging or discharging state, the charging and discharging power of the system is zero. Equation (13), respectively, represents the energy level constraint and dynamic energy transfer constraint of the energy storage system; equation (14) indicates that the energy storage system cannot be charged and discharged at the same time during operation.

2.2.3. Interval with Grid. The power grid serves as the energy supply in the grid-connected microgrid and is the most important component of maintaining the stability of the power grid.

$$
\begin{gathered}
\delta_{\text {grid }}^{\text {in }}(k) P_{\text {grid }}^{\text {in, min }} \leq P_{\text {grid }}^{\text {in }}(k) \leq \delta_{\text {grid }}^{\text {in }}(k) P_{\text {grid }}^{\text {in, max }}, \\
\delta_{\text {grid }}^{\text {out }}(k) P_{\text {grid }}^{\text {out, min }} \leq P_{\text {grid }}^{\text {out }}(k) \leq \delta_{\text {grid }}^{\text {out }}(k) P_{\text {grid }}^{\text {out, max }}, \\
\delta_{\text {grid }}^{\text {in }}(k)+\delta_{\text {grid }}^{\text {out }}(k) \leq 1 .
\end{gathered}
$$

Equations (16) and (17) are the minimum and maximum power constraints for interaction with the large power grid. Equation (18) restricts the purchase and sale of electricity at any time.

The prerequisite for the microgrid to achieve stable and reasonable dispatch is that the microgrid system can maintain stable and reliable operation at all times. This requires the microgrid to be able to ensure the balance of supply and demand within the system at all times, which is also one of the important constraints of the microgrid which is as follows:

$$
\begin{aligned}
P_{\text {grid }}^{\text {in }}(k) & +P_{\text {solar }}(k)+P_{\text {bess }}^{d}(k)=P_{\text {grid }}^{\text {out }}(k)+\sum_{i=1}^{N_{\mathrm{EV}}} P_{\mathrm{EV}, i}(k) \\
& +P_{\text {bess }}^{c}(k)+P_{\text {load }}(k) .
\end{aligned}
$$

In the formula, the left side represents the power purchased, the power of photovoltaic power generation, and the discharge power of the energy storage system, which are the power supply of the microgrid system. The right sideshows the selling power, electric vehicle charging power, energy storage system charging power, and public load power in turn. 


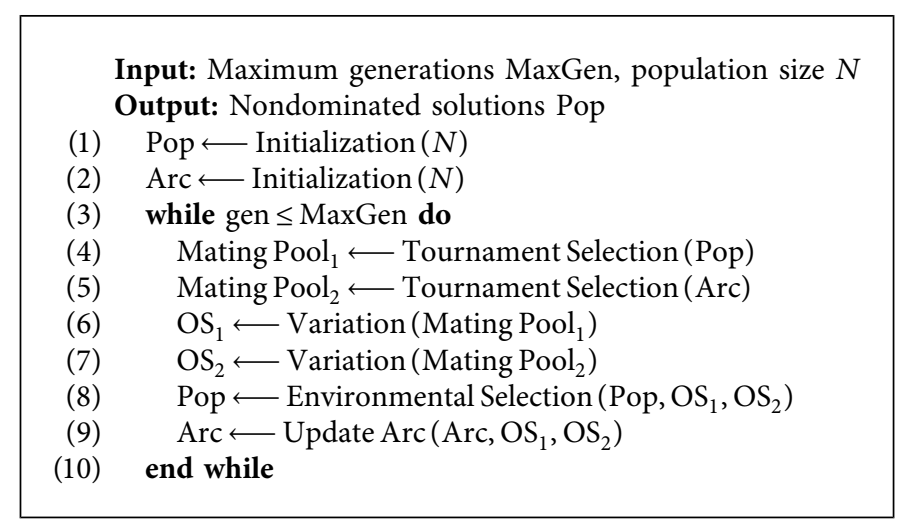

Algorithm 1: General framework.

\section{Archive-Based Multiobjective Evolutionary Algorithm}

There are many approaches to deal with constraints in a multiobjective optimization problem (MOP) [12]. These approaches can obtain favorable results on benchmark problems. However, real-world engineering problems are generally complex and multimodal. Generally, the feasible regions of these problems are usually separated from each other in objective space and decision space.

To address this issue, we proposed an archived-based constrained evolutionary multiobjective optimization algorithm, termed ACEMO, which introduces an archive to help explore the whole decision space. The thoughts of two-archive can be widely found in some state-of-the-art algorithms [13], which is proved effective and efficient. Specifically, we do not process the constraints in the introducing archive. This archive aims to find the Pareto optimal front of a normal MOP without considering the constraints. Therefore, solutions in the archive will quickly converge to the true Pareto front. In the beginning of the evolution process, solutions in the main population and the archive are randomly generated. As the algorithm runs, solutions in main population will converge to the optimal without constraints, which is similar to that of traditional constrained EMO. As a result, the main population will utilize the information provided by the archive and help jump out of the local optimal. The framework of the proposed algorithm is explained in Algorithm 1.

As we can see from Algorithm 1, the main population and the introduced archive will coevolve by sharing their offspring (line 8-9). Therefore, solutions can be added to the main population and help to converge to the truePareto front.

\section{Experiment}

4.1. Experimental Setting. This paper selects a microgrid demonstration project in a certain scenic spot as the research object for analysis and research, and its simplified model is shown in Figure 1. In order to verify the effectiveness of the algorithm, we chose the SRA algorithm [14], which has outstanding performance in handling constraint problems, as a comparison. The population size of the algorithm is set to 100 , the number of iterations is set to 800 , and the probability of crossover and mutation is set to 0.8 and 0.1 , respectively. In order to reduce the influence of random factors, we carried out the experiment 31 times and displayed the result that was closest to the average value. Other related parameters are set in Table 1.

The microgrid in this scenic spot is connected to the external power grid and belongs to the grid-connected microgrid. The system includes an energy storage system (accessible or not can be controlled), photovoltaic power generation equipment, public loads, and public charging piles. There are a total of 15 charging piles provided in the scenic spot, which can provide charging services for tourists' electric vehicles. The maximum charging power that can be provided is $7 \mathrm{~kW}$, the charging efficiency is 0.95 , and the energy self-loss is $0.02 \mathrm{~kW}$. The maximum energy storage capacity of the energy storage equipment is $70 \mathrm{kWh}$, the charge and discharge efficiency is 0.95 , the self-discharge energy loss of the energy storage system is $0.02 \mathrm{~kW}$, and the maximum interactive power is $80 \mathrm{~kW}$.

4.2. Result Analysis. Figure 2 shows the Pareto frontier of the ACEMO and SRA algorithm on the charging station microgrid problem. It can be seen from the figure that the front edge obtained by the ACEMO algorithm is closer to the origin of the coordinates, which means that the results obtained by the ACEMO algorithm have better convergence. At the same time, it can be seen from the figure that the Pareto front obtained by the SRA algorithm performs poorly in terms of distribution and uniformity. This is because the ACEMO algorithm introduces archive, which can explore the entire decision space without considering the constraints of the problem, and the probability of jumping out of the local optimum is greatly improved.

In order to better display the optimization results of the algorithm, we need to choose a solution from many Pareto fronts. Generally speaking, if the decision maker can give his preference information for this problem, such as the weight value between different goals, he can calculate the weighted objective function and take the smallest solution as the optimal solution. However, it is often difficult for decision makers to give their preference value. Therefore, some 


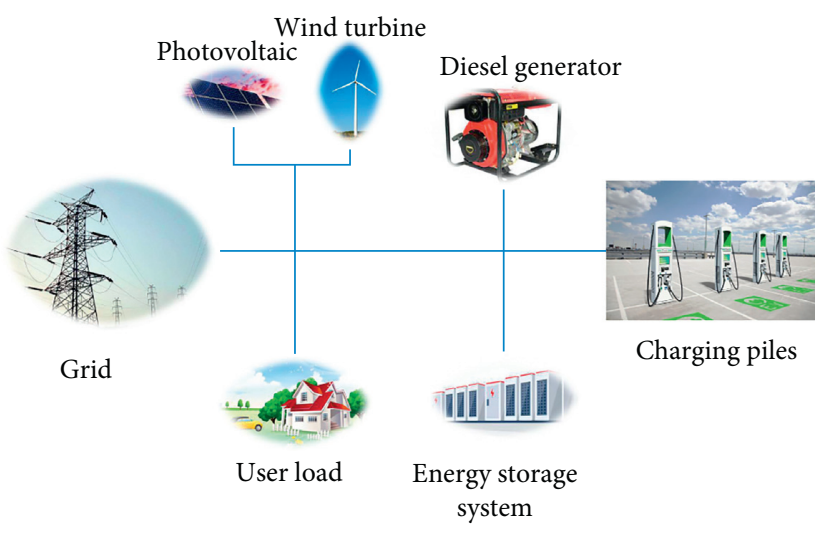

FiguRE 1: Illustration of a microgrid.

TABle 1: Parameter settings.

\begin{tabular}{lc}
\hline Parameter name & Data \\
\hline Battery capacity $(\mathrm{kWh})$ & 57 \\
Maximum power $(\mathrm{kW})$ & 90 \\
Pure electric driving kilometers $(\mathrm{km})$ & 316 \\
Charging time $(\mathrm{h})$ & 7 \\
\hline
\end{tabular}

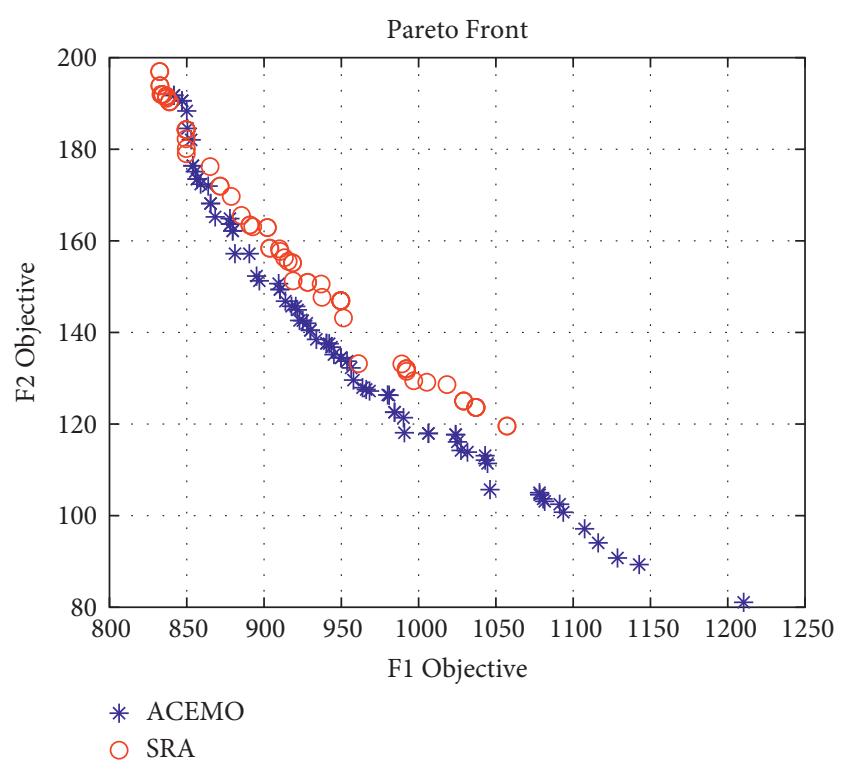

Figure 2: The Pareto front of ACEMO and SRA on microgrid problem.

auxiliary decision-making methods are particularly important, such as TOPSIS [15], gray correlation analysis [16], and knee method [3,17]. For simplicity, we used the TOPSIS method for selection in this article.

After confirming the optimal solution, we analyzed the details of the solution. The operating status of each component of the charging station microgrid is shown in Figure 3. From the figure, we can see that the renewable energy power generation peaked at noon $(10: 00-14: 00)$, and during this process, the energy storage system began to

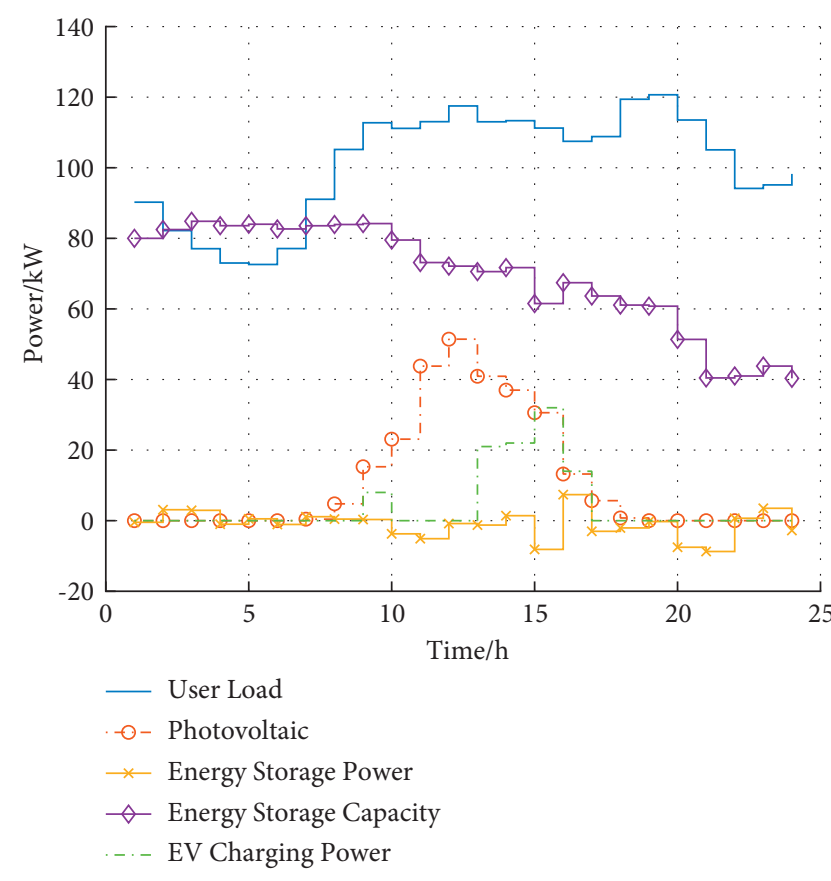

FIgURE 3: The running status of microgrid components.

charge in a plan to make rational use of the excess electric energy. Since the peak time for electric vehicles arrives at around $15: 00$, the peak time for electric vehicle charging is also after this. Through the control of the charging pile and the energy storage system, it can be found that the excess electric energy has been efficiently used, and the daily operating costs of the charging station microgrid have also been reduced.

The experimental results verify the effectiveness of our proposed algorithm, and its performance on the microgrid problem is better than SRA. In addition, the correctness and validity of the charging station microgrid model proposed in this paper can be verified by analyzing the operating status of each component of the microgrid.

\section{Conclusion}

With the extensive development and use of renewable energy sources, a large number of new devices continue to emerge, which puts forward new requirements for microgrid energy management planning. Although the current research on energy management of microgrid has achieved certain results, there are still considerable problems in solving the uncertainty problem. In this paper, after considering the random arrival of electric vehicles, the corresponding research on the energy management of the charging station microgrid is carried out, and the relevant mathematical model is established. Secondly, this paper proposes a multiobjective optimization algorithm that deals with constraints to solve the model. Through experiments, the effectiveness of the model and solution method proposed in this paper is verified. The study also pointed out that the reasonable management of electric vehicle charging can greatly reduce the load fluctuation of the large power grid, 
improve the stability of the power grid, and reduce the operating cost of the microgrid at the charging station.

This paper considers the stochastic process of electric vehicles to establish a microgrid energy management model and verifies its feasibility through experiments. However, this article still has deficiencies and needs to be improved in the following aspects. The first is that the scale of electric vehicles is small. Although it can reflect the actual results to a certain extent, as more and more electric vehicles are put into use, the scale of the problem will be further expanded. Secondly, electric vehicles are a very special and flexible mobile energy storage system. This article only considers the use of electric vehicles as mobile loads, without considering its potential advantages as mobile energy storage. In the next work, we will focus on the above issues and conduct more indepth research.

\section{Data Availability}

The data used to support the findings of this study are included within the article.

\section{Conflicts of Interest}

The authors declare that they have no conflicts of interest.

\section{Acknowledgments}

This work was supported by Science and Technology Project of SGCC: "Research on Typical Design and Operation Service of Urban Smart Energy System".

\section{References}

[1] B. Simanic, B. Nordquist, H. Bagge, and D. Johansson, "Predicted and measured user-related energy usage in newly built low-energy schools in Sweden," Journal of Building Engineering, vol. 29, Article ID 101142, 2020.

[2] W. Huang, D. Zheng, X. Chen et al., "Standard thermodynamic properties for the energy grade evaluation of fossil fuels and renewable fuels," Renewable Energy, vol. 147, pp. 21602170, 2020.

[3] W. Li, G. Zhang, X. Yang, Z. Tao, and H. Xu, "Sizing a hybrid renewable energy system by a coevolutionary multiobjective optimization algorithm," Complexity, vol. 2021, Article ID 8822765, 9 pages, 2021.

[4] W. Li, T. Zhang, and R. Wang, "Energy management model of charging station micro-grid considering random arrival of electric vehicles," in Proceedings of the 2018 IEEE International Conference on Energy Internet (ICEI), pp. 29-34, IEEE, Beijing, China, May 2018.

[5] G. Putrus, P. Suwanapingkarl, D. Johnston, E. Bentley, and M. Narayana, "Impact of electric vehicles on power distribution networks," in Proceedings of the 2009 IEEE Vehicle Power and Propulsion Conference, pp. 827-831, IEEE, Dearborn, Michigan, September 2009.

[6] W. Li, R. Wang, T. Zhang, M. Ming, and H. Lei, "Multiscenario microgrid optimization using an evolutionary multiobjective algorithm," Swarm and Evolutionary Computation, vol. 50, Article ID 100570, 2019.

[7] E. S. Rigas, S. D. Ramchurn, and N. Bassiliades, "Managing electric vehicles in the smart grid using artificial intelligence: a survey," IEEE Transactions on Intelligent Transportation Systems, vol. 16, no. 4, pp. 1619-1635, 2014.

[8] X. Fang, D. Yang, and G. Xue, "Evolving smart grid information management cloudward: a cloud optimization perspective," IEEE Transactions on Smart Grid, vol. 4, no. 1, pp. 111-119, 2013.

[9] T. Zhang, W. Chen, Z. Han, and Z. Cao, "Charging scheduling of electric vehicles with local renewable energy under uncertain electric vehicle arrival and grid power price," IEEE Transactions on Vehicular Technology, vol. 63, no. 6, pp. 2600-2612, 2013.

[10] B. Zhang, Y. Sun, B. Li, and J. Li, "A modeling method for the power demand of electric vehicles based on Monte Carlo simulation," in Proceedings of the 2012 Asia-Pacific Power and Energy Engineering Conference, pp. 1-5, IEEE, Shanghai, China, March 2012.

[11] H. Zhao, Q. Wu, S. Hu, H. Xu, and C. N. Rasmussen, "Review of energy storage system for wind power integration support," Applied Energy, vol. 137, pp. 545-553, 2015.

[12] A. Slowik and H. Kwasnicka, "Evolutionary algorithms and their applications to engineering problems," Neural Computing \& Applications, vol. 32, no. 16, pp. 12363-12379, 2020.

[13] G. Peng and K. Wolter, "A novel archive maintenance for adapting weight vectors in decomposition-based multi-objective evolutionary algorithms," in Proceedings of the 2020 IEEE Congress on Evolutionary Computation (CEC), pp. 1-8, IEEE, Wellington, New Zealand, June 2020.

[14] M. A. Jan, M. Sagheer, H. U. Khan et al., "Hybrid stochastic ranking for constrained optimization," IEEE Access, vol. 8, pp. 227270-227287, 2020.

[15] P. Chen, "Effects of the entropy weight on topsis," Expert Systems with Applications, vol. 168, Article ID 114186, 2021.

[16] L. Zhu, C. Zhao, and J. Dai, "Prediction of compressive strength of recycled aggregate concrete based on gray correlation analysis," Construction and Building Materials, vol. 273, Article ID 121750, 2021.

[17] W. Li, R. Wang, T. Zhang, M. Ming, and K. Li, "Reinvestigation of evolutionary many-objective optimization: focus on the pareto knee front," Information Sciences, vol. 522, pp. 193-213, 2020. 